\title{
Evaluation of the accuracy and sensitivity of high-throughput sequencing technology using known microbiota
}

\author{
FANJING MENG $^{1 *}$, TINGTAO CHEN $^{1 *}$, XIN WANG $^{1}$, XIAOLEI WANG $^{1}$, HUA WEI $^{2}$, \\ PUYUAN TIAN $^{2}$, HUAN WANG $^{1}$, XIAOXIAO ZHAO ${ }^{1}$, LIANG SHEN $^{3}$ and HONGBO XIN ${ }^{1}$ \\ ${ }^{1}$ Institute of Translational Medicine, Nanchang University, Nanchang, Jiangxi 330031; ${ }^{2}$ State Key Laboratory of \\ Food Science and Technology, Nanchang University, Nanchang, Jiangxi 330047; ${ }^{3}$ Department of Obstetrics and Gynaecology, \\ Shandong Provincial Hospital Affiliated to Shandong University, Jinan, Shandong 250012, P.R. China
}

Received April 6, 2017; Accepted September 9, 2017

DOI: $10.3892 / \mathrm{mmr} .2017 .7849$

\begin{abstract}
Next generation sequencing provides an excellent platform to explore microbiota in any given environment, and little work is required to evaluate the accuracy and sensitivity of high-throughput sequencing technology. In the present study, a known microbiota containing Escherichia coli, Lactobacillus plantarum, Streptococcus thermophilus, Bifidobacterium bifidum, Bacillus subtilis, Enterococcus faecalis and Salmonella typhimurium was used to evaluate the high-throughput sequencing technology. The results suggested that there were 122.7 operational taxonomic units (OTUs) in all groups, which is 17.5 -fold (the whole OTU number/the actual bacterial number) greater compared with the actual microbial number in each group, and the Venn method indicated that only $46.38 \%$ (64/138), 58.70\% (81/138), 86.13\% (118/137), 83.57\% $(117 / 140)$ and $89.29 \%(125 / 140)$ of the common OTUs were identified in groups A, B, C, D and E, of which the majority of OTUs did not belong to known bacteria. In addition, the DNA extraction and amplification efficiency failed to identify bacteria at the phylum, class, order, family, genus and species levels, which may further increase false information of microbial analysis. In conclusion, the present study provided basic datato investigate the potential drawbacks of high-throughput sequencing technology, which will help researchers to avoid exaggerating the bacterial number when this technology is applied to study microbiota in particular environments.
\end{abstract}

Correspondence to: Professor Tingtao Chen or Professor Hongbo Xin, Institute of Translational Medicine, Nanchang University, 1299 Xuefu Road, Nanchang, Jiangxi 330031, P.R. China E-mail: chentingtao1984@163.com

E-mail: hongboxin@yahoo.com

${ }^{*}$ Contributed equally

Key words: high-throughput sequencing, accuracy and sensitivity, microbiota, E. coli, $\beta$ diversity

\section{Introduction}

Previously, only a small fraction of all bacteria had been isolated and characterized, and analysis of any bacterial community was severely limited by the available technology and the shortage of reference genomes $(1,2)$. The development of next generation sequencing provided an excellent platform to explore the association between humans and microbiota (3-6).

The human body harbors bacterial, viral and eukaryotic communities in the skin, nasopharynx, oral cavity, respiratory tract, gastrointestinal tract and female reproductive tract (7-10), and the microbes have profound implications on humanmetabolism, immunity and the gut-brain axis (3-6). As it has been demonstrated, the human intestine exists in symbiosis with hundreds of trillions of microbes, and there is an increasing awareness that the bacteria residing within the gut has a significant influence on host health (7). In addition, microbial dysbiosis caused by the altered intestinal microbes has been proved to contribute to the onset of several disorders, and it is important to identify the key bacteria that serve a direct or indirect effect on human health.

Previously, analysis of microbial diversity in specific environments was severely limited by available technologies and referenced genomes. Technological advances in next generation sequencing have enabled the elucidation of the pleiotropic effects of microorganisms on the human host, and the high-throughput sequencing can detect almost all the DNA signatures of microbes within specific environments, even those bacteria present in low numbers (2). However, a number of studies have only focused on the examination of microbial diversity using high-throughput sequencing technology, which provided little information on the potential and limitations of this approach in microbial ecology studies (11-13).

Sample handling, DNA extraction, amplification efficiency, run processing and downstream analyses have seriously affected the generation of high quality data (2,14-17). Therefore, in the present study, a number of known bacteria were mixed together at a certain percentage, and extracted DNAs were subjected high throughput sequencing to evaluate the accuracy and sensitivity of high-throughput sequencing technology, providing basic data to help researchers to better 
investigate the relationship between microorganisms and host health.

\section{Materials and methods}

Bacterial activation and culture. A total of seven common bacterial species, including Escherichia coli (no. 44102; isolated from the donated human feces to screen the bacteria in human intestines, and stored in the authors' lab), Lactobacillus plantarum (no. HM218749; isolated from sourdough and stored in the author's lab), Streptococcus thermophilus [no. 19258; American Type Culture Collection (ATCC), Manassas, VA, USA], Bifidobacterium bifidum (no. WBIN03; isolated from yogurt and stored in the authors' lab), Bacillus subtilis (no. 14416; ATCC), Enterococcus faecalis (no. HM218543; isolated from the donated human feces to screen the bacteria in human intestines, and stored in the authors' lab) and Salmonella typhimurium (no. 14028; ATCC), were selected in the present studyand divided into five groups (Table I). All bacteria were propagated in corresponding media three times before DNA extraction (Table I).

Extraction of genomic DNA and high-throughput sequencing. Genomic DNA from each sample was extracted using a TIANamp Genomic DNA kit (Tiangen Biotech Co., Ltd., Beijing, China) combined with bead beating, as previously described (3). Subsequently, the genomic DNA was sent to a high-throughput sequencing company (Biomarker Technologies Corporation, Beijing, China) for high-throughput sequencing and analysis.

The extracted genomic DNAs extracted from these seven common bacteria were used as the templates, and the universal primer pair 338F/806R with the respective barcode (supplied by BiomarkerTechnologiesCorporation, Beijing, China) for ease of identification (Table II) were used to to amplify the V3-V4 region of 16S ribosomal (r)RNA genes of all samples. Polymerase chain reaction (PCR), pyrosequencing of the PCR amplicons and quality control of raw data were performed as described previously with minor modifications as presented in the 'Bioinformatics and multivariate statistics' section below (18).

Bioinformatics and multivariate statistics. Low-quality sequences were eliminated from the analysis based on the following criteria: i) Raw reads shorter than $400 \mathrm{bp}$; ii) a sequence producing $>8$ homopolymers; iii) $>2$ mismatches in the primers; and iv) 1 or more mismatches in the barcode. Pyrosequenced amplicons were removed using the PyroNoise algorithm in Mothur (version 1.33.3) (19). Bioinformatics analysis was implemented using the Quantitative Insights Into Microbial Ecology (QIIME) platform (version 1.8.0) (20). Briefly, 16S rRNA operational taxonomic units (OTUs) were clustered using an open-reference OTU picking protocol based on $97 \%$ nucleotide similarity with the UCLUST algorithm (21). ChimeraSlayer was employed to remove chimeric sequences (22). The relative abundance of each OTU was determined as a proportion of the sum of sequences for each sample. Taxonomic relative abundance profiles (including at the phylum, class, order, family and genus levels) were generated based on OTU annotation. The microbial community structure (i.e., species richness, evenness and between-sample diversity) of bacterial samples was estimated by biodiversity. Shannon index, phylogenetic diversity, chaol index and the observed number of species were used to evaluate $\alpha$ diversity, and the weighted and unweighted UniFrac distances were used to evaluate $\beta$ diversity.

All of these indices ( $\alpha$ and $\beta$ diversity) were calculated via the QIIME pipeline.

Statistical analysis. Statistical analysis was implemented using the R platform. Principal coordinate analysis (PCoA) was performed using the 'ape' package based on the UniFrac distances between samples. The difference among groups was further assessed using analysis of similarities and multi-response permutation planning methodswith Metastats software (http://metastats.cbcb.umd.edu/) as described previously, and statistical significance was set at $\mathrm{P}<0.05$ for correction of multiple comparisons (23).

\section{Results}

Sequencing coverage. To compare the microbes in each sample, 16S rRNA amplicon sequencing analysis was used to sequence the V3-V4 hypervariable region, the sequencing data was filtered to obtain the valid data, and the effective tags of all samples were clustered, and those sequences with $>97 \%$ similarity were considered to beone OTU. In total, 2,032,484 filtered clean tags $(135,498.9$ tags/sample) and 1,840 OTUs were obtained from all the samples with an average of 122.7 OTUs per group (Table III). The chaol index was almost saturated and the rarefaction curve of every sample could enter the plateau phase (data not shown).

Shared genera in each sample. The Venn diagrams reflected the differences between all groups. As presented in Fig. 1, there were 138 (A), 138 (B), 137 (C), 140 (D) and 140 (E) OTUs in each group, and the percentage of common OTUs was 46.38 (64/138), 58.70 (81/138), 86.13 (118/137), 83.57 (117/140) and $89.29 \%(125 / 140)$, respectively.

$\beta$ diversity of the microbial community. The overall graph of the microbial composition of the samples was obtained by using PCoA, based on the relative abundance profiles of bacterial taxa. As presentedin Fig. 2, the samples A1 and A3 clustered together on the right upper corner of the coordinate axis, samples A2, B1, B3, C1, C2, C3, D1, D2, D3, E1, $\mathrm{E} 2$ and $\mathrm{E} 3$ gathered together on the lower right corner of the coordinate axis and sample B2 scattered on the bottom left corner.

Scientific classification of bacterial communities in each sample. To further investigate the relative abundance of the known bacteria in each group, the identified bacterial abundance was compared at the phylum, class, order, family, genus and species levels. Fig. 3 demonstrated that all the known bacteria were detected at the phylumand class levels, although their percentages only occupied 41.66 and $28.10 \%$ of the total bacteria, respectively. In addition, certain bacteria, for example, Bifidobacteriales at the order level, Streptococcaceae and Bifidobacteriaceae at the family level, 
Table I. Bacterial composition in groups A, B, C, D and E.

Group, $\mathrm{CFU} / \mathrm{ml}$

\begin{tabular}{lccccc}
\cline { 2 - 5 } Bacteria & $\mathrm{A}$ & $\mathrm{B}$ & $\mathrm{C}$ & $\mathrm{D}$ & $\mathrm{E}$ \\
\hline E. coli (44102) & $1 \times 10^{6}$ & $1 \times 10^{4}$ & $1 \times 10^{3}$ & $1 \times 10^{2}$ & $1 \times 10$ \\
L. plantarum (HM218749) & $1 \times 10^{6}$ & $1 \times 10^{4}$ & $1 \times 10^{3}$ & $1 \times 10^{2}$ & $1 \times 10$ \\
S. thermophilus (ATCC 19258) & $1 \times 10^{6}$ & $1 \times 10^{6}$ & $1 \times 10^{6}$ & $1 \times 10^{6}$ & $1 \times 10^{6}$ \\
B. bifidum (WBIN03) & $1 \times 10^{6}$ & $1 \times 10^{6}$ & $1 \times 10^{6}$ & $1 \times 10^{6}$ & $1 \times 10^{6}$ \\
B. subtilis (ATCC 14416) & $1 \times 10^{6}$ & $1 \times 10^{6}$ & $1 \times 10^{6}$ & $1 \times 10^{6}$ & $1 \times 10^{6}$ \\
E. faecalis (HM218543) & $1 \times 10^{6}$ & $1 \times 10^{6}$ & $1 \times 10^{6}$ & $1 \times 10^{6}$ & $1 \times 10^{6}$ \\
S. typhimurium (ATCC 14028) & $1 \times 10^{6}$ & $1 \times 10^{6}$ & $1 \times 10^{6}$ & $1 \times 10^{6}$ & $1 \times 10^{6}$ \\
\hline
\end{tabular}

CFU, colony-forming units.

Table II. Primer barcodes.

\begin{tabular}{ll}
\hline No. & \multicolumn{1}{c}{ Sequence } \\
\hline A1 & AGGGTCAATGAACCTT \\
A2 & AGGGTCAAAGTCAACA \\
A3 & AGGGTCAACTCTCTAT \\
B1 & AGGGTCAAAGAGTAGA \\
B2 & AGGGTCAAGTAAGGAG \\
B3 & AGGGTCAAACTGCATA \\
$C 1$ & AGGGTCAAAAGGAGTA \\
$C 2$ & AGGGTCAACTAAGCCT \\
$C 3$ & AGGAGTGGTGAACCTT \\
$D 1$ & AGGAGTGGAGTCAACA \\
$D 2$ & AGGAGTGGCTCTCTAT \\
$D 3$ & AGGAGTGGAGAGTAGA \\
E1 & AGGAGTGGGTAAGGAG \\
E2 & AGGAGTGGACTGCATA \\
E3 & AGGAGTGGAAGGAGTA
\end{tabular}

Streptococcus and Bifidobacterium at the genus level, and E. coli, L. plantarum, S. thermophilus, B. bifidum, B. subtilis and $S$.typhimurium at the species level, failed to be detected, and the identified bacteria only occupied 20.84, 20.98, 19.31 and $1.62 \%$ at the order, family, genus and species levels, respectively (Fig. 3).

Relative abundance of the bacterial communities in each sample. To compare the relative abundances among different groups, the Metastats method was applied in the presentstudy. At the genus level, the relative abundances of Salmonella, Bacillus and Enterococcus were each lowered or enhanced in the groups, although their actual number in each group was the same (Table I). For E. coli, actual bacterial number in groups A, B, C, D and E, was $1 \times 10^{6}, 1 \times 10^{4}, 1 \times 10^{3}, 1 \times 10^{2}$ and $1 \times 10^{1} \mathrm{CFU} / \mathrm{ml}$, respectively, while the OTU numbers in groups $\mathrm{C}, \mathrm{D}$ and $\mathrm{E}$ were significantly higher compared with groups A and B (Fig. 4). In addition, though the OTU number of L. plantarum in groups B, C, D and E was significantly decreased compared with group A (Fig. 4), there was no significant difference observed among groups B, C, D and E, and the actual bacterial number of $L$. plantarum in groups B, $\mathrm{C}, \mathrm{D}$ and $\mathrm{E}$ was $1 \times 10^{4}, 1 \times 10^{3}, 1 \times 10^{2}$ and $1 \times 10^{1}$, respectively (Table I).

\section{Discussion}

The world is dominated by prokaryotes. The total number of microbial cells on Earth is estimated to be $1 \times 10^{30}$ and, in the human body alone, there are up to 100 trillion organisms, which approximately equates to ten times the number of our own human cells $(24,25)$, and there are millions of prokaryotic species which may not be cultivated $(15,26)$. In the humanbody, bacteria serve important roles in the modulation of digestive, endocrineand immune functions. With the discovery of more recent culture-independentsequencing-based methods, the composition and diversity of the human microbiome is being uncovered. However, sample handling, DNA extraction, amplification efficiency, run processing and downstream analyses may seriously affect the generation of high quality data $(2,14-17)$ and, therefore, it is important to evaluate the accuracy and sensitivity of high-throughput sequencing technology.

In the present study, seven common bacteria were mixed together to make a known microbiota, of which $S$. thermophilus, B. bifidum, B. subtilis, E. faecalis and S. typhimurium sustained a constant number of $1 \times 10^{6} \mathrm{CFU} / \mathrm{ml}$ in each sample, and the typical gram-negative bacteria $E$. coli and gram-positive bacteria $L$. plantarum were decreased between groups A and E. Following mixing of the known bacteria, DNA extraction was performed and the extracted genomic DNA was used as a template to amplify the V3-V4 region of $16 \mathrm{~S}$ rRNA genes using primers of $338 \mathrm{~F} / 806 \mathrm{R}$ (18), and the results suggested that the DNA quality and PCR amplicons met the requirements for pyrosequencing (data not shown).

To evaluate tag quality, the raw tags and clean tags, average bp and OTUs per sample were compared. The mean number of 135,498.9 clean tags, average length of $467.7 \mathrm{bp}$, and saturated chaol index and rarefaction curves ensured their reliability for future analysis. However, the average OTUs (122.7) in all groups indicated a 17.5-fold (122.7/7) increase compared with 
Table III. Number of raw tags, clean tags, average bp, OTUs and actual bacterial composition in groups A, B, C, D and E by high-throughput sequencing.

\begin{tabular}{|c|c|c|c|c|c|}
\hline Sample ID & Raw tags & Clean tags & Average length, bp & OTU & Actual bacterial number \\
\hline A1 & 180,452 & 163,786 & 467 & 120 & 7 \\
\hline A2 & 167,355 & 147,971 & 467 & 82 & 7 \\
\hline A3 & 159,440 & 144,734 & 467 & 120 & 7 \\
\hline B1 & 135,586 & 118,439 & 467 & 131 & 7 \\
\hline B2 & 88,832 & 71,781 & 469 & 84 & 7 \\
\hline B3 & 134,956 & 117,370 & 468 & 136 & 7 \\
\hline $\mathrm{C} 1$ & 139,230 & 123,136 & 468 & 122 & 7 \\
\hline $\mathrm{C} 2$ & 163,421 & 144,130 & 467 & 130 & 7 \\
\hline $\mathrm{C} 3$ & 188,147 & 167,154 & 468 & 131 & 7 \\
\hline D1 & 187,451 & 162,980 & 468 & 132 & 7 \\
\hline D2 & 139,141 & 120,711 & 468 & 128 & 7 \\
\hline D3 & 147,408 & 129,402 & 467 & 129 & 7 \\
\hline E1 & 147,574 & 125,254 & 468 & 135 & 7 \\
\hline E2 & 166,163 & 147,835 & 468 & 131 & 7 \\
\hline E3 & 165,512 & 147,801 & 468 & 129 & 7 \\
\hline Average & $154,044.5$ & $135,498.9$ & 467.7 & 122.7 & 7 \\
\hline
\end{tabular}

OTU, operational taxonomic units.

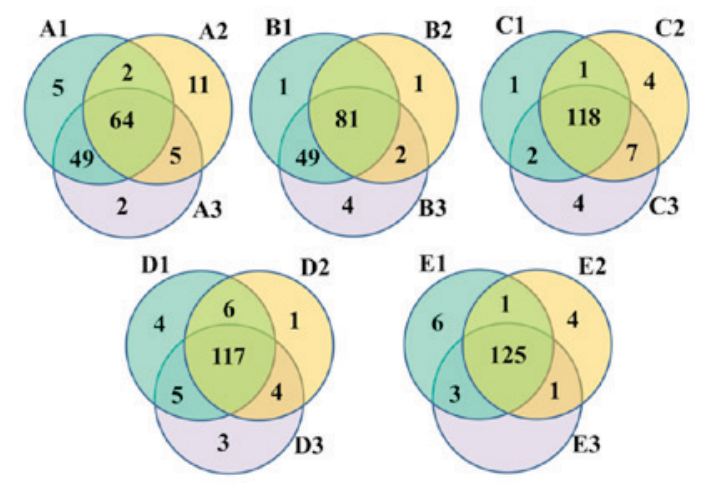

Figure 1. Scalar-Venn representation of the microbiota among groups A, B, $\mathrm{C}, \mathrm{D}$ and $\mathrm{E}$.

the actual microbial number in each group (7). To evaluate the consistency of the high-throughput sequencing, the Venn and PCoA methods were utilized and the results suggested that only $46.38 \%(64 / 138), 58.70 \%(81 / 138), 86.13 \%(118 / 137)$, $83.57 \%(117 / 140)$ and $89.29 \%$ (125/140) common OTUs were identified in groups A, B, C, D and E, of which the majority of OTUs did not belong to the added bacteria. However, the PCoA results demonstrated that the majority of samples clustered together, except for sample B2.

Compared with the relative abundance of known microbiota, all known bacteria were identified at the phylum and class levels; one or more bacteria was missed at the order, family and genus levels, and the known bacteria only occupied $\sim 20 \%$ of the total OTUs at these levels. As microbiota are generally analyzed at the genus level, the statistical analysis in the present study was performed at the genus level, and Salmonella, Bacillus and Enterococcus, which existed at the

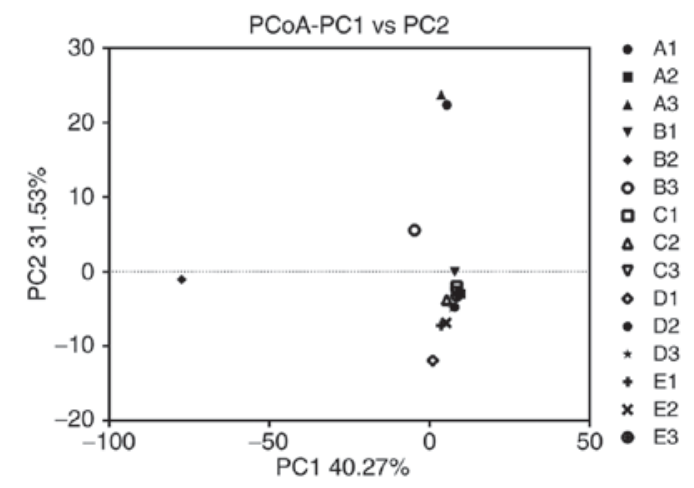

Figure 2. PCoA of $\beta$ diversity index of groups A, B, C, D and E. PCoA, principle component analysis.

same number in each group, exhibited a significant decreased or increase using high-throughput sequencing technology; whereas, the ten-fold dilution of Escherichia and Lactobacillus among groups C, D and E exhibited little alteration.

Microbial genomic DNA extraction and purification is the first step for library preparation however researchers indicated that there were significant differences in microbial composition when comparing microbiota diversity obtained from the same samples using different DNA extraction methods (27). To avoid the influence of DNA extraction, all the DNA samples were simultaneously extracted by the same researcher using the same DNA extraction kit. Therefore, the DNA extraction method is not the key factor for the misidentification of the known microbiota. In addition, all the DNA was amplified using the same primers of $338 \mathrm{~F} / 806 \mathrm{R}$, as a result of the potential for amplification bias during PCR amplification reactions and the generation of chimeric amplification products 

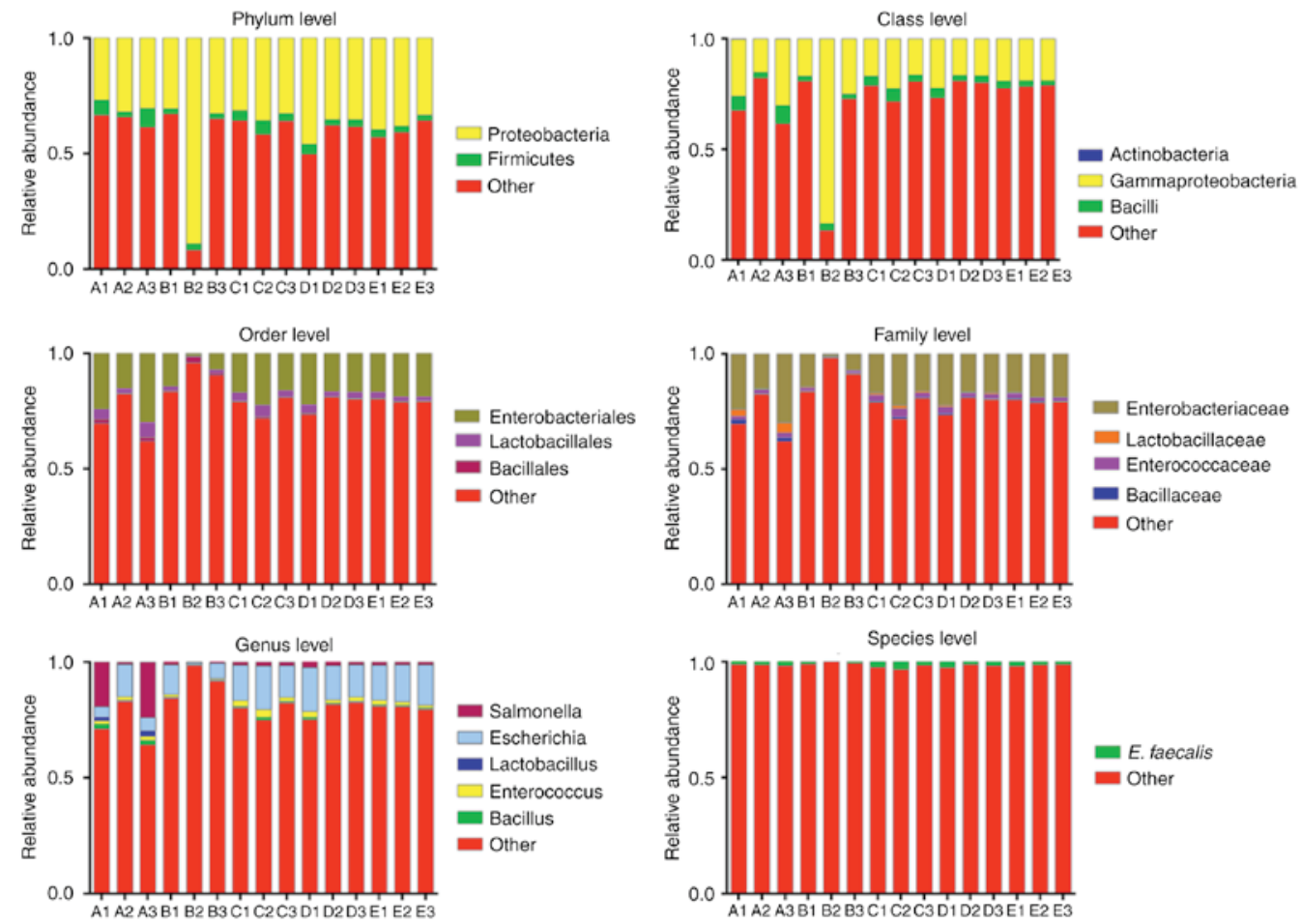

Figure 3. Ratios of the known bacteria in groups A, B, C, D and E at the phylum, class, order, family, genus and species levels.
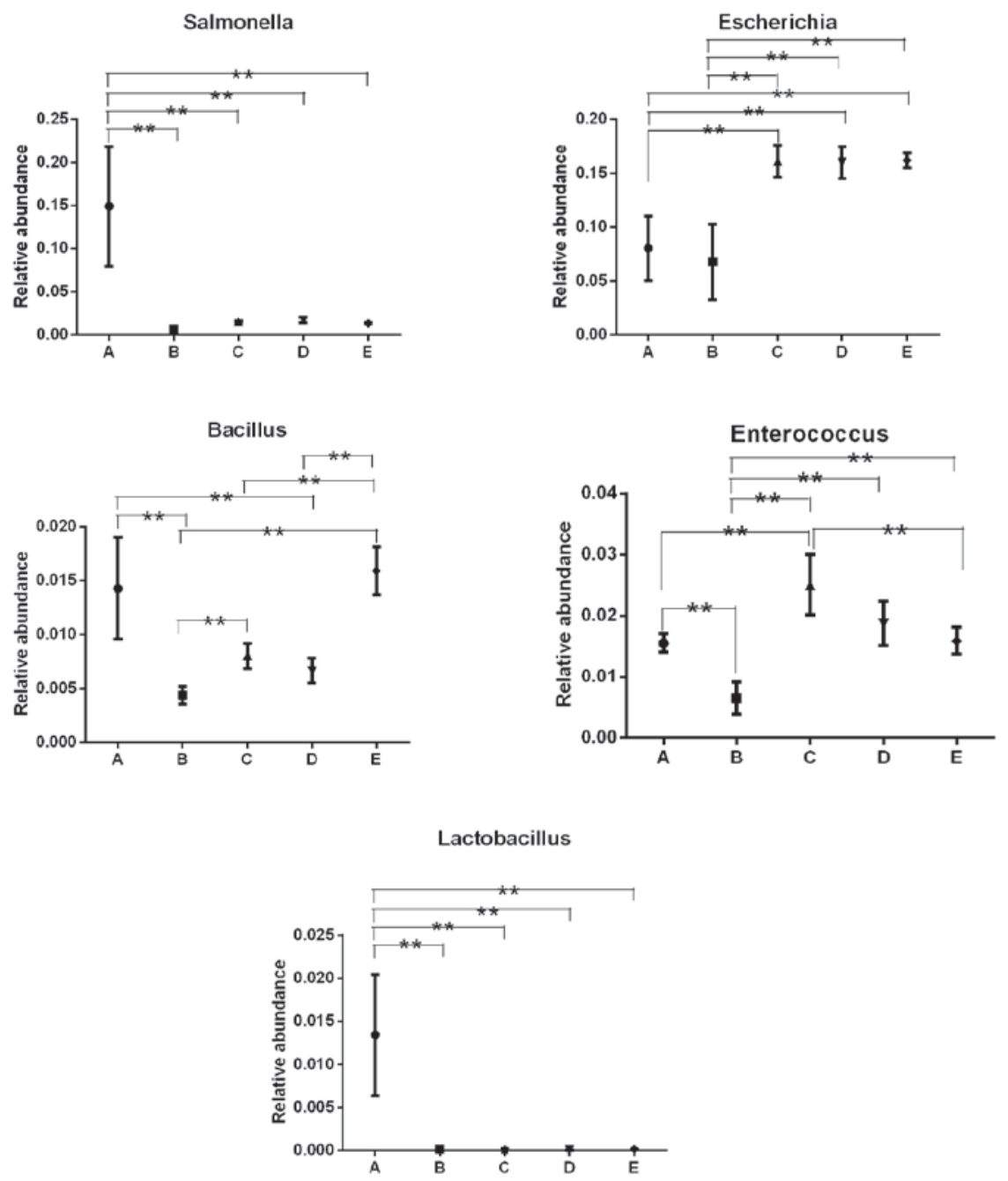

Figure 4. Relative microbial abundance of Bacillus, Enterococcus, Escherichia, Lactobacillus and Salmonella in groups A, B, C, D and E.* P $<0.05$ vs. group A. 
that may exaggerate the bacterial number (28). Furthermore, the chimeric sequences, which are not identified by computational filtering software will lead to incorrect taxonomic identifications and an overestimated bacterial richness in the final microbiota profiling results $(24,28)$.

In conclusion, the results of the present study suggested that the actual bacterial number in a specific environment maybe greatly exaggerated due to run processing and downstream analyses, and that DNA extraction and amplification efficiency may cause a reduction or exaggeration of certain bacteria. Therefore, certain measures, for example, adding the indicated bacteria/microbiota and analysing using more than three types of calculation software, are required to provide reasonable results. However, this result was only based on the present study, and the small size and lack of the comparison among various sequencing companies may not allowthe results of the present study to fully reflect the actual drawbacks of high-throughput sequencing. In the authors' future work, the microbial diversity in various environments will be tested using enlarged sample sizes and comparing the data quality among different sequencing companies. This will provide basic data for the improvement of high-throughput sequencing technology and benefit its applications in the monitoring of bacterial alterations during various diseases.

\section{Acknowledgements}

The present study was supported by grants from the National Natural Science Foundation of China (grant nos. 31560264, 81503364, 91639106, 81270202, and 91339113), the National Basic Research Program of China (grant no. 2013CB531103) and grants from Jiangxi Province (grant nos. 20171BCB23028 and 20175526).

\section{References}

1. Shokralla S, Spall JL, Gibson JF and Hajibabaei M Next-generation sequencing technologies for environmental DNA research. Mol Ecol 21: 1794-1805, 2012.

2. Yue-Xin M, Holmstrom C and Webb J: Application of denaturing gradient gel electrophoresis (DGGE) in microbial ecology. Acta Ecologica Sinica 23: 1561-1569, 2003.

3. Yu X, Wu X, Qiu L, Wang D, Gan M, Chen X, Wei H and $\mathrm{Xu}$ F: Analysis of the intestinal microbial community structure of healthy and long-living elderly residents in Gaotian village of Liuyang City. Appl Microbiol Biot 99: 9085-9095, 2015.

4. Sivan A, Corrales L, Hubert N, Williams JB, Aquino-Michaels K, Earley ZM, Benyamin FW, Lei YM, Jabri B, Alegre ML, et al: Commensal Bifidobacterium promotes antitumor immunity and facilitates anti-PD-L1 efficacy. Science 350: 1084-1089, 2015.

5. Zhernakova A, Kurilshikov A, Bonder MJ, Tigchelaar EF, Schirmer M, Vatanen T, Mujagic Z, Vila AV, Falony G, Vieira-Silva $S$, et al: Population-based metagenomics analysis reveals markers for gut microbiome composition and diversity. Science 352: 565-569, 2016.

6. Derrien M and van Hylckama Vlieg JE: Fate, activity, and impact of ingested bacteria within the human gut microbiota. Trends Microbiol 23: 354-360, 2015

7. Mimee M, Citorik RJ and Lu TK: Microbiome therapeutics-Advances and challenges. Adv Drug Deliver Revi 105 44-54, 2016.
8. Chen T, Shi Y, Wang X, Wang X, Meng F, Yang S, Yang J and Xin H: High-throughput sequencing analyses of oral microbial diversity in healthy people and patients with dental caries and periodontal disease. Mol Med Rep 16: 127-132, 2017.

9. Fang X, Wang X, Yang S, Meng F, Wang X, Wei H and Chen T: Evaluation of the microbial diversity in amyotrophic lateral sclerosis using high-throughput sequencing. Front Microbiol 7: 1479, 2016.

10. Wang X, Hu X, Deng K, Cheng X, Wei J, Jiang M, Wang X and Chen T: High-throughput sequencing of microbial diversity in implant-associated infection. Infect Genet Evol 43: 307-311, 2016.

11. Planý M, Šoltys K, Budiš J, Mader A, Szemes T, Siekel P and Kuchtaa T: Potential of high-throughput sequencing for broad-range detection of pathogenic bacteria in spices and herbs. Food Control 70: 360-370, 2016.

12. Ishaq SL and Wright AD: High-throughput DNA sequencing of the ruminal bacteria from moose (Alces alces) in Vermont, Alaska, and Norway. Microb Ecol 68: 185-195, 2014.

13. Hamilton MJ, Weingarden AR, Unno T, Khoruts A and Sadowsky MJ: High-throughput DNA sequence analysis reveals stable engraftment of gut microbiota following transplantation of previously frozen fecal bacteria. Gut Microbes 4: 125-135, 2013.

14. Moorthie S, Mattocks CJ and Wright CF: Review of massively parallel DNA sequencing technologies. Hugo J 5: 1-12, 2011.

15. Kircher M, Heyn P and Kelso J: Addressing challenges in the production and analysis of illumina sequencing data. BMC Genomics 12: 382, 2011.

16. Kircher M: Understanding and improving high-throughput sequencing data production and analysis: 225, 2011.

17. Hall N: Advanced sequencing technologies and their wider impact in microbiology. J Exp Biol 210: 1518-1525, 2007.

18. Xu J, Lian F, Zhao L, Zhao Y, Chen X, Zhang X, Guo Y, Zhang C, Zhou Q, Xue Z, et al: Structural modulation of gut microbiota during alleviation of type 2 diabetes with a Chinese herbal formula. Isme J 9: 552-562, 2015.

19. Schloss PD, Westcott SL, Ryabin T, Hall JR, Hartmann M, Hollister EB, Lesniewski RA, Oakley BB, Parks DH, Robinson CJ, et al: Introducing mothur: Open-source, platform-independent, community-supported software for describing and comparing microbial communities. Appl Environ Microb 75: 7537-7541, 2009.

20. Caporaso JG, Kuczynski J, Stombaugh J, Bittinger K, Bushman FD, Costello EK, Fierer N, Peña AG, Goodrich JK, Gordon JI, et al: QIIME allows analysis of high-throughput community sequencing data. Nat Methods 7: 335-336, 2010.

21. Davenport M, Poles J, Leung JM, Wolff MJ, Abidi WM, Ullman T, Mayer L, Cho I and Loke P: Metabolic alterations to the mucosal microbiota in inflammatory bowel disease. Inflamm Bowel Dis 20: 723-731, 2014.

22. Haas BJ, Gevers D, Earl AM, Feldgarden M, Ward DV, Giannoukos G, Ciulla D, Tabbaa D, Highlander SK, Sodergren E, et al: Chimeric 16S rRNA sequence formation and detection in Sanger and 454-pyrosequenced PCR amplicons. Genome Res 21: 494-504, 2011.

23. Lu K, Ryan PA, Schlieper KA, Graffam ME, Levine S, Wishnok JS, Swenberg JA, Tannenbaum SR and Fox JG: Arsenic exposure perturbs the gut microbiome and its metabolic profile in mice: An integrated metagenomics and metabolomics analysis. Environ Health Perspect 122: 284, 2014.

24. Boers SA, Jansen R and Hays JP: Suddenly everyone is a microbiota specialist. Clin Microbiol Infec 22: 581-582, 2016.

25. Di Bella JM, Bao Y, Gloor GB, Burton JP and Reid G: High throughput sequencing methods and analysis for microbiome research. J Microbiol Meth 95: 401-414, 2013.

26. Van Vliet AH: Next generation sequencing of microbial transcriptomes: Challenges and opportunities. Fems Microbiol Lett 302: 1-7, 2010.

27. Kennedy NA, Walker AW, Berry SH, Duncan SH, Farquarson FM, Louis P, Thomson JM; UK IBD Genetics Consortium, Satsangi J, Flint HJ, et al: The impact of different DNA extraction kits and laboratories upon the assessment of human gut microbiota composition by $16 \mathrm{~S}$ rRNA gene sequencing. PLoS One 9: e88982, 2014.

28. Boers SA, Hays JP and Jansen R: Micelle PCR reduces chimera formation in 16S rRNA profiling of complex microbial DNA mixtures. Sci Rep 5: 14181, 2015. 